\title{
DISCOVERY OF A STRONGLY LENSED MASSIVE QUIESCENT GALAXY AT $z=2.636$ : SPATIALLY RESOLVED SPECTROSCOPY AND INDICATIONS OF ROTATION
}

\author{
Andrew B. Newman ${ }^{1}$, Sirio Belli ${ }^{2,3}$, and Richard S. Ellis ${ }^{2,4}$ \\ ${ }^{1}$ The Observatories of the Carnegie Institution for Science, 813 Santa Barbara St., Pasadena, CA 91101, USA; anewman@obs.carnegiescience.edu \\ ${ }^{2}$ Department of Astrophysics, California Institute of Technology, MS 249-17, Pasadena, CA 91125, USA \\ ${ }^{3}$ Max-Planck-Institut für extraterrestrische Physik (MPE), Giessenbachstrasse 1, D-85748 Garching, Germany \\ ${ }^{4}$ European Southern Observatory (ESO), Karl-Schwarzschild-Strasse 2, D-85748 Garching, Germany \\ Received 2015 September 16; accepted 2015 October 12; published 2015 October 23
}

\begin{abstract}
We report the discovery of RG1M0150, a massive, recently quenched galaxy at $z=2.636$ that is multiply imaged by the cluster MACSJ0150.3-1005. We derive a stellar mass of $\log M_{*}=11.49_{-0.16}^{+0.10}$ and a half-light radius of $R_{e \text {, maj }}=1.8 \pm 0.4 \mathrm{kpc}$. Taking advantage of the lensing magnification, we are able to spatially resolve a remarkably massive yet compact quiescent galaxy at $z>2$ in ground-based near-infrared spectroscopic observations using Magellan/FIRE and Keck/MOSFIRE. We find no gradient in the strength of the Balmer absorption lines over $0.6 R_{e}-1.6 R_{e}$, which are consistent with an age of $760 \mathrm{Myr}$. Gas emission in [N II] broadly traces the spatial distribution of the stars and is coupled with weak $\mathrm{H} \alpha$ emission $(\log [\mathrm{N}$ II] $/ \mathrm{H} \alpha=0.6 \pm 0.2)$, indicating that $\mathrm{OB}$ stars are not the primary ionizing source. The velocity dispersion within the effective radius is $\sigma_{e \text {, stars }}=271 \pm 41 \mathrm{~km} \mathrm{~s}^{-1}$. We detect rotation in the stellar absorption lines for the first time beyond $z \sim 1$. Using a two-integral Jeans model that accounts for observational effects, we measure a dynamical mass of $\log M_{\text {dyn }}=11.24 \pm 0.14$ and $V / \sigma=0.70 \pm 0.21$. This is a high degree of rotation considering the modest observed ellipticity of $0.12 \pm 0.08$, but it is consistent with predictions from dissipational merger simulations that produce compact remnants. The mass of RG1M0150 implies that it is likely to become a slowly rotating elliptical. If it is typical, this suggests that the progenitors of massive ellipticals retain significant net angular momentum after quenching which later declines, perhaps through accretion of satellites.
\end{abstract}

Key words: galaxies: elliptical and lenticular, $\mathrm{cD}$ - galaxies: kinematics and dynamics gravitational lensing: strong

\section{INTRODUCTION}

Observations are beginning to reveal the bulk properties of the high-redshift progenitors of massive elliptical galaxies. Massive quiescent galaxies are present at $z>2$ but have very compact sizes compared to local early-type galaxies (ETGs). Much effort in recent years has focused on understanding the growth of these "nuggets" and the emergence of extended stellar envelopes (e.g., Trujillo et al. 2006; van Dokkum et al. 2010; Newman et al. 2012; Patel et al. 2013; Belli et al. 2015). Recent observations are also beginning to link these early quiescent galaxies to star-forming progenitors at even earlier epochs via their masses, sizes, and kinematics (Barro et al. 2014; Toft et al. 2014; van Dokkum et al. 2015).

Numerical simulations have made increasingly detailed predictions for the properties of ETGs formed in disk mergers (e.g., Cox et al. 2006; Robertson et al. 2006; Hopkins et al. 2009; Zolotov et al. 2015). Forming a dense stellar core requires significant dissipation, and such gas-rich mergers are predicted to produce rotating remnants-possibly with a velocity exceeding the bulk of today's ETGs (Wuyts et al. 2010). Different channels for the formation of compact galaxies can be tested with stellar population gradients (Wellons et al. 2015) and kinematic data. However, such comparisons require spatially resolved observations, which are very challenging given the small angular sizes of typical $z>2$ quiescent galaxies $(\sim 0$ ". $1-0$ "' 2$)$. Gravitational lensing provides a promising route toward resolving these compact systems. The main challenge in locating lensed examples is the need for wide-area near-infrared (NIR) imaging to overcome their rarity and faintness at optical wavelengths. Thus far only three example have been published (Muzzin et al. 2012; Geier et al. 2013), and none has spatially resolved spectroscopic data.

In this Letter we present the discovery of RG1M0150, a rare example of a massive, quiescent galaxy at $z=2.636$ that is multiply imaged by the cluster MACSJ0150.3-1005 (R.A. $1^{\mathrm{h}} 50^{\mathrm{m}} 21^{\mathrm{s}}$. 3, decl. $-10^{\circ} 05^{\prime} 30^{\prime \prime}, z=0.365$; Ebeling et al. 2001). RG1M0150 was located in a search for bright sources with red NIR colors that are magnified by clusters in the Hubble Space Telescope $(H S T)$ archive. It is extremely bright $\left(K_{s, \mathrm{AB}}=19.2\right)$ and well suited for detailed follow-up observations. We analyze NIR spectra collected with Magellan/FIRE and Keck/ MOSFIRE and are able to spatially resolve stellar absorption lines with spectral resolution sufficient for kinematic work. We use these unique data to study the resolved stellar populations and rotational support of RG1M0150.

Throughout we adopt $\mathrm{AB}$ magnitudes and the cosmological parameters $\left(\Omega_{m}, \Omega_{v}, h\right)=(0.3,0.7,0.7)$.

\section{OBSERVATIONS}

\subsection{Imaging}

RG1M0150 was identified in a search of archival HST/ WFC3 images of massive clusters. We targeted bright, magnified sources with red NIR colors indicative of a Balmer/4000 $\AA$ break at $z \gtrsim 2$. HST imaging of RG1M0150 covers WFC3/F140W, WFC3/F110W, ACS/F814W, and WFCP2/F606W (SNAP-12884 and SNAP-11103, P. I. Ebeling; see Figure 1). We also obtained $J, H$, and $K_{s}$ band observations with Magellan/FourStar (Persson et al. 2013) which were reduced following procedures discussed by 

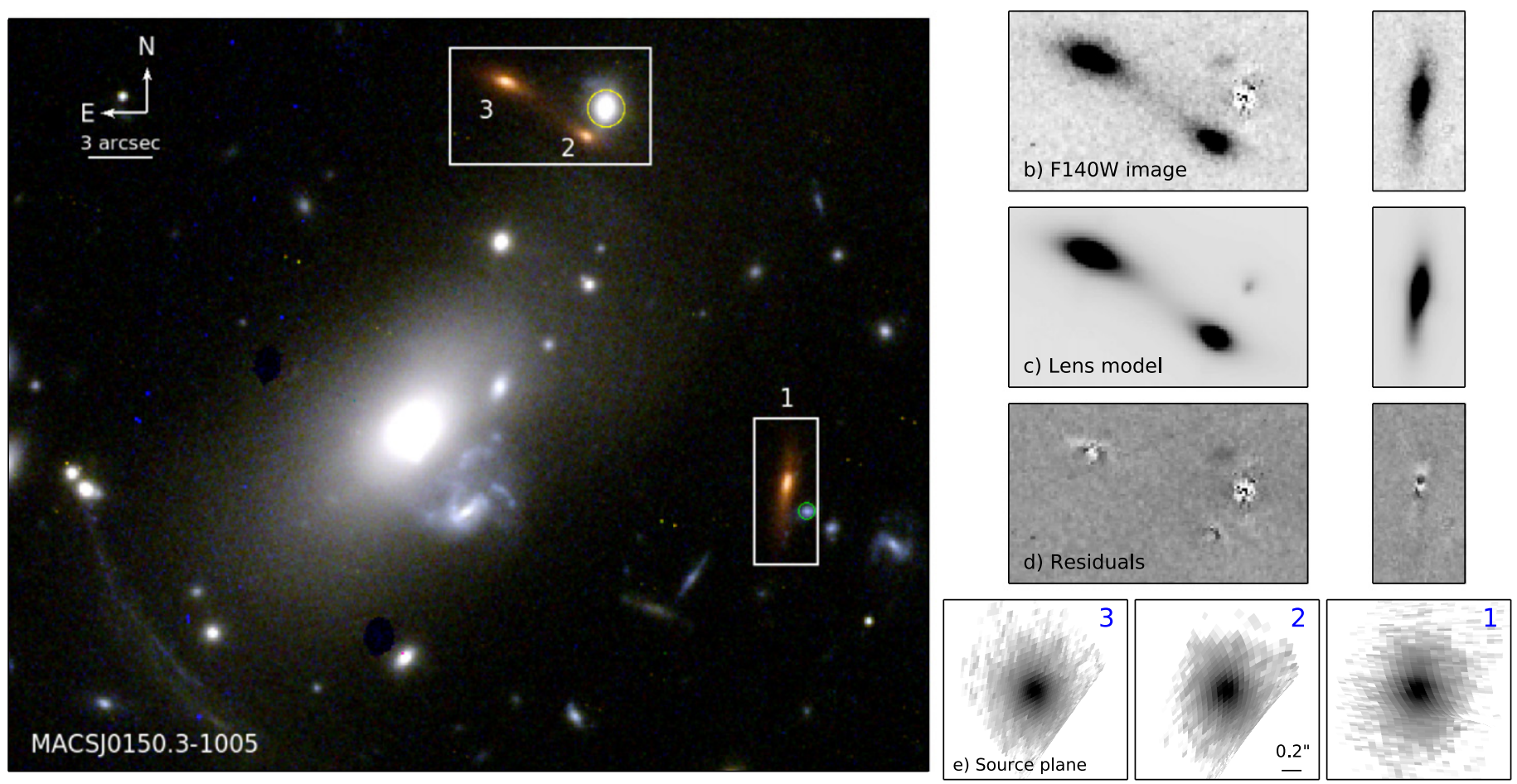

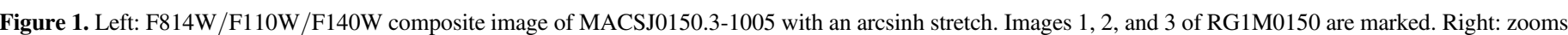

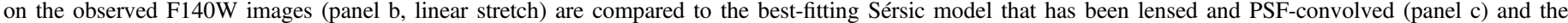

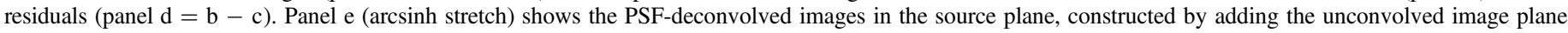
model to the residuals (i.e., Data - Model $_{\text {convolved }}+\mathbf{M o d e l}_{\text {unconvolved }}$ ) and casting pixels to the source plane.

Kelson et al. (2014). Colors were measured in a $0{ }^{\prime \prime} 6 \times 0$ "' 75 rectangular aperture along Image 1 matching the spectroscopic aperture used in Section 2.2. Matching of the point-spread function (PSF) between the FourStar and HST images was performed as described by Newman et al. (2012). Total fluxes were obtained by scaling to the F140W flux in larger $8^{\prime \prime} \times 2$ " 5 rectangle. We also incorporate 3.4 and $4.6 \mu \mathrm{m}$ measurements from the AllWISE catalog. ${ }^{5}$ Zeropoint and PSF matching uncertainties of $0.03 \mathrm{mag}$ (HST), $0.05 \mathrm{mag}$ (FourStar), and 0.1 mag (WISE) were added in quadrature.

\subsection{Spectroscopy}

We obtained NIR spectroscopic observations of Image 1 of RG1M0150 with both MOSFIRE (McLean et al. 2012) at the Keck I telescope and FIRE (Simcoe et al. 2013) at the Magellan Baade telescope. FIRE is a cross-dispersed echellette instrument that covers $0.8-2.45 \mu \mathrm{m}$ simultaneously. The MOSFIRE data cover the $H$ band only but afford somewhat cleaner sky subtraction owing to the faster dithering cadence. A position angle (PA) of $-7^{\circ} .5$ was selected to place the slit along Image 1.

The MOSFIRE exposures total 260 minutes taken over 2014 November 26-27, consisting of 2 minute integrations in an $\mathrm{AB}$ dither pattern. The 0.'7 slit provided a resolution of $\sigma_{\text {inst }}=36 \mathrm{~km} \mathrm{~s}^{-1}$. Another slit was positioned on a star to measure the average seeing of 0. " 67 . Data were reduced using the MOSFIRE Data Reduction Pipeline.

The FIRE observations were conducted over 2014 November 1 and 3 and total 390 minutes, consisting of 30 minute exposures using up-the-ramp sampling. The $6^{\prime \prime} \times 0$ !"75 slit

\footnotetext{
http://wise2.ipac.caltech.edu/docs/release/allwise/
}

provided a resolution of $\sigma_{\text {inst }}=30 \mathrm{~km} \mathrm{~s}^{-1}$. The average seeing of 0 ." 55 was determined by monitoring a star on the NIR slit viewing camera. The data were reduced using a modified version of the FIREHOSE pipeline that produces twodimensional rectified spectra. For both data sets, telluric absorption correction and flux calibration were performed using A0V star observations and xtellcor (Vacca et al. 2003).

\subsection{Lens Model}

We construct a lens model using the three images of RG1M0150 as constraints. Analytic models describe the lens mass distribution and the source light profile. Their parameters are constrained simultaneously using the ray-tracing code described in Newman et al. (2015), which fits the pixel-level WFC3/F140W data.

Given its smooth, symmetric appearance, the source was modeled using an elliptical Sérsic profile. To model the cluster lens, we use several dual pseudo-isothermal elliptical (dPIE) mass distributions (see Elíasdóttir et al. 2007). Each is described by two scale radii $r_{\text {cut }}$ and $r_{\text {core }}$ and a normalization $\sigma_{0}$, in addition to its center, PA, and ellipticity. All parameters of the dPIE describing the cluster dark matter halo are free other than $r_{\text {cut }}$, which is beyond the strong lensing zone and can be fixed to $1 \mathrm{Mpc}$ (e.g., Richard et al. 2010). Additional dPIE components account for deflection by luminous cluster galaxies and are generally modeled using the scaling relations $\sigma_{0}=\sigma_{0}^{\star}\left(L / L_{*}\right)^{1 / 4}$ and $r_{\text {cut }}=r_{\text {cut }}^{\star}\left(L / L_{*}\right)^{1 / 2}$, with priors on $\sigma_{0}^{\star}$ and $r_{\text {cut }}^{\star}$ as described by Newman et al. (2013). However, the brightest cluster galaxy and the two galaxies circled in Figure 1, which are important perturbers, were allowed to depart from these scaling relations. We also included external shear to fine 

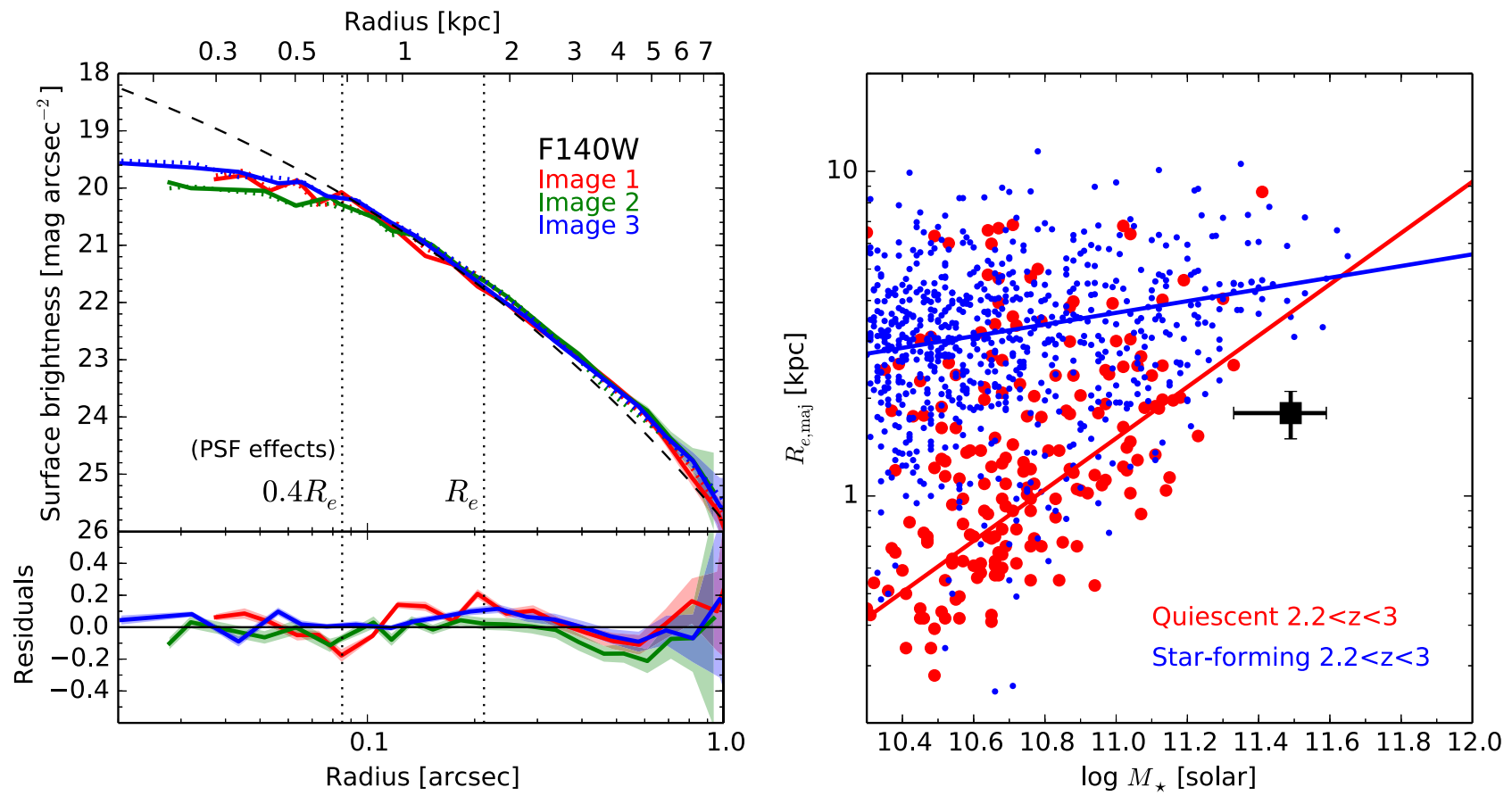

Figure 2. Left: source plane F140W surface brightness profiles reconstructed from each image of RG1M0150 (solid colored lines). Dotted colored lines show the corresponding reconstructions from the PSF-convolved Sérsic model fits, with residuals indicated in the lower panel. (At small radii, the reconstructions differ because the PSF affects each image differently.) Shading indicates the $1 \sigma$ uncertainty. The black dashed line shows the unconvolved Sérsic model. Right: the compact size of RG1M0150 (black) is compared to $2.2<z<3$ field galaxies drawn from the 3D-HST survey catalogs (Skelton et al. 2014) with structural parameters from van der Wel et al. (2014).

tune the image reconstruction, although this did not make a major difference to our final model.

Figure 1 demonstrates that our best-fitting model produces three images that match the F140W data well. The model produces areal magnifications of $\mu_{1}=3.9_{-0.7}^{+1.6}, \mu_{2}=2.4_{-0.5}^{+1.5}$, and $\mu_{3}=4.4_{-0.8}^{+2.0}$ for Images 1,2 , and 3, respectively. Uncertainties were conservatively estimated by comparing to an independent set of Lenstool models (Kneib et al. 1993; Jullo et al. 2007) constrained only by the positions, ellipticity, and fluxes of the images and varying the mass model components.

\section{STRUCTURE AND STELLAR POPULATION}

\subsection{Stellar Structure}

Our best-fitting Sérsic model indicates that RG1M0150 is a compact, nearly round, concentrated galaxy with an effective radius $R_{e, \text { maj }}=0$ "' $23 \pm 0$ ". $05=1.8 \pm 0.4 \mathrm{kpc}$ (semimajor axis), Sérsic index $n=3.5 \pm 0.9$, magnitude $m_{\mathrm{F} 140 \mathrm{~W}}=21.8_{-0.2}^{+0.4}, \quad$ axis $\quad$ ratio $\quad b / a=0.88 \pm 0.06, \quad$ and $\mathrm{PA}=2^{\circ} \pm 14^{\circ}$. Uncertainties were estimated from the scatter in parameters obtained when fitting the three multiple images individually ( $m_{\mathrm{F} 140 \mathrm{~W}}$ also includes the systematic magnification uncertainty).

We reconstruct source plane surface brightness profiles for each image in Figure 2. The lensing magnification enables us to probe down to scales of $R=700 \mathrm{pc}=0.4 R_{e}$ before PSF blurring becomes significant. Over the range $R=0.4 R_{e}-4 R_{e}$ a single Sérsic profile remains adequate to fit the F140W light with systematic residuals of $\lesssim 10 \%$ (lower panel). The restframe colors of RG1M0150 $(U-V=1.69 \pm 0.06$, $V-J=0.73 \pm 0.14$ ) satisfy the criterion often used to select quiescent galaxies. The right panel of Figure 2 demonstrates that RG1M0150 is a compact galaxy, lying slightly below the mass-size relation defined by other quiescent systems at similar redshift.

\subsection{Stellar Population}

RG1M0150 presents a red continuum shape with strong Balmer absorption dominating the rest-optical spectrum. In Figure 3 we combine the broadband photometry with our FIRE spectrum to constrain the stellar population using our pyspecfit code (Newman et al. 2014). We adopt solar metallicity Bruzual \& Charlot (2003) models and a Chabrier (2003) initial mass function. The FIRE spectrum is extracted in a rectangular aperture extending \pm 0 " 6 from the center of Image 1, which best approximates $\pm R_{e}$ in the source plane.

We infer a remarkably high stellar mass of $\log M_{*}=11.49_{-0.16}^{+0.10}$, scaled to the source plane Sérsic model flux and including the magnification uncertainty. Given the very high indicated mass, we simulate the probability distribution of the most massive $2<z<3$ quiescent galaxy lensed by a cluster in the HST/WFC 3 archive (80 clusters, assuming a typical cross-section $\sigma=200 \operatorname{arcsec}^{2}$ for magnification $\mu>3$ similar to MACSJ0150.3-1005) using the Muzzin et al. (2013) mass functions. A lensed galaxy with $\log M_{*}>11.49$ is indeed quite rare $(p=0.005)$. The probability increases to $p=0.02$ if the threshold is lowered by $1 \sigma$ to $\log M_{*}>11.33$. If we take our simulated probability distribution as a prior in order to account for an Eddington-type bias, the posterior for the stellar mass would shift downward to $\log M_{*}=11.33_{-0.15}^{+0.11}$.

For an exponentially declining star formation history, we find an age of $760 \pm 50 \mathrm{Myr}$ and a prompt $e$-folding time of 97 \pm 11 Myr. The corresponding ongoing star formation rate is 

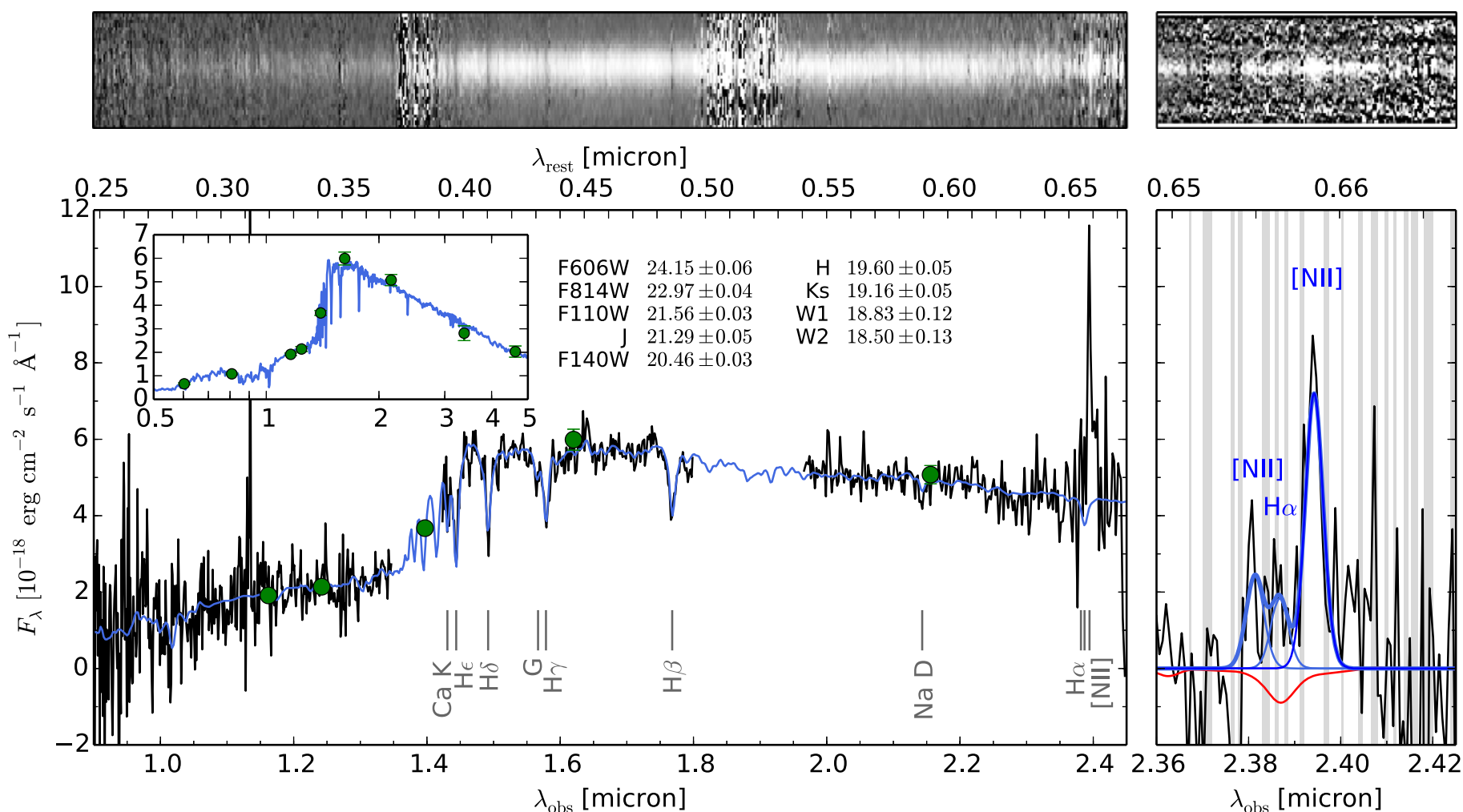

Figure 3. Left: the FIRE spectrum of Image 1, resampled to $300 \mathrm{~km} \mathrm{~s}^{-1}$ bins for clarity, is compared to the stellar population model described in Section 3.2. Green points show the broadband photometry, which is displayed on an expanded scale in the inset. Resampled two-dimensional spectra are shown above. Right: zoom on the $\mathrm{H} \alpha+[\mathrm{N}$ II] region with the stellar model (red) subtracted. Blue lines show a Gaussian decomposition with [N II] $] \lambda 585 /[\mathrm{N}$ II $] \lambda 6550$ fixed to 3 . The observed spectrum has been scaled by a factor of 3.6 to match the total flux of Image 1. Gray bands denote strong sky lines.
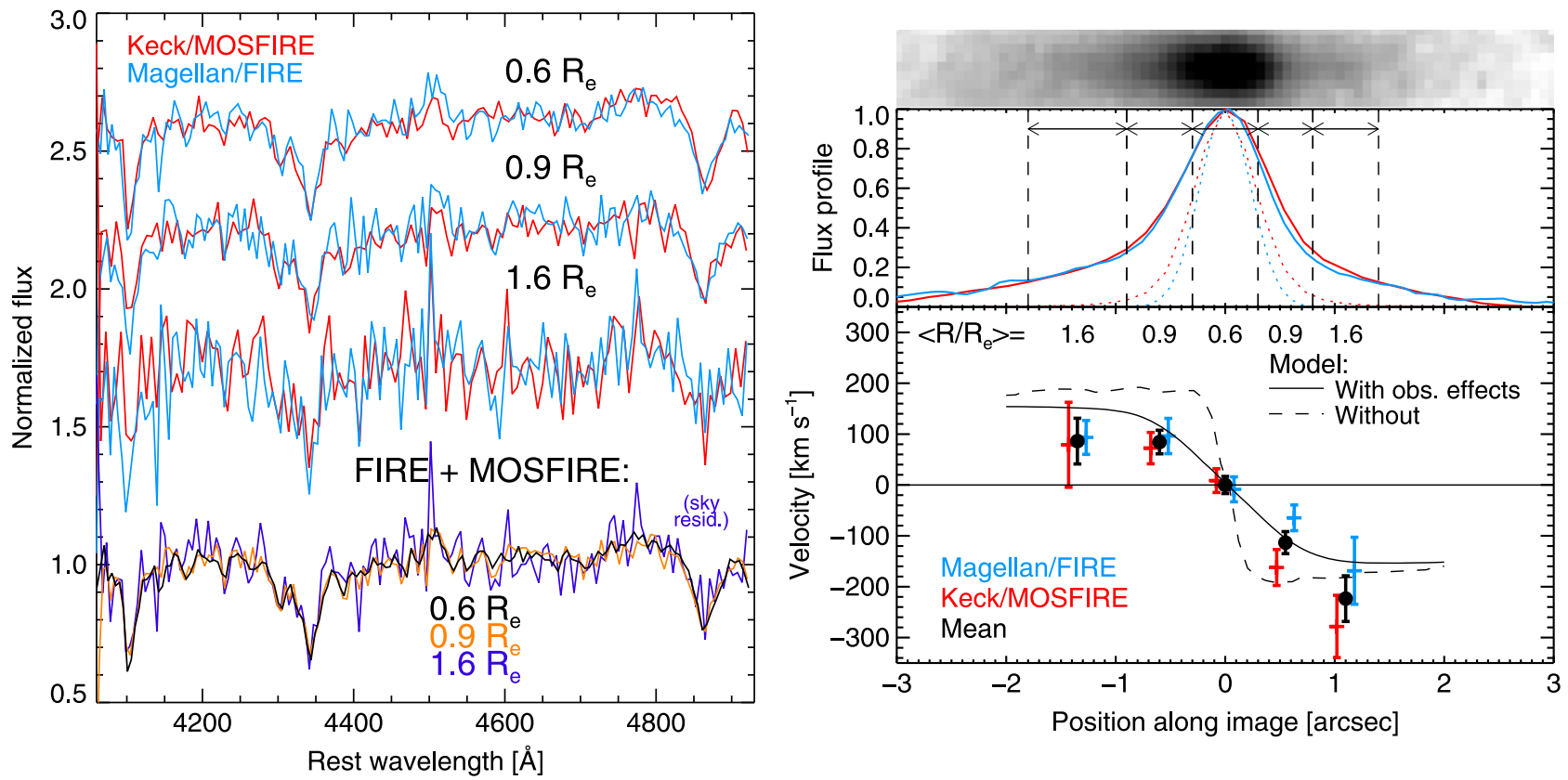

Figure 4. Left: resolved Balmer absorption lines extracted in several spatial bins. Corresponding spectra on opposite sides of the galaxy center have been shifted to the systemic redshift and coadded. The FIRE and MOSFIRE spectra are combined in the lower spectrum and demonstrate no significant gradients in Balmer line strength over $0.6 R_{e}-1.6 R_{e}$. Right: the top panel illustrates the position of the spectrograph slit as seen in the WFC3/F140W image. The middle panel compares the flux distributions in the spectra to the PSFs (dotted), demonstrating that we spatially resolve Image 1. Five extraction bins are indicated. The lower panel indicates the presence of rotation measured using the stellar absorption lines.

only $\mathrm{SFR}=2 \pm 1 M_{\odot} \mathrm{yr}^{-1}$ (accounting for magnification), indicating that star formation was recently and quickly truncated in this galaxy. The specific star formation rate is $\log \mathrm{SSFR} / \mathrm{yr}^{-1}<-11.1(68 \% \mathrm{CL})$ independent of magnification. Dust attenuation is moderate with $A_{V}=0.52 \pm 0.08$ assuming the Calzetti et al. (2000) reddening curve. (These 
uncertainties are purely statistical and do not include known differences among stellar population models.)

The lensing magnification presents a unique opportunity to probe the location of the last star formation event by spatially resolving the Balmer absorption. We extract spectra in 5 rectangular apertures whose boundaries along the spectroscopic slit are chosen to examine the same luminosity-weighted radius in the source plane on either side of the galaxy: $\langle R\rangle=0.6 R_{e}$, $0.9 R_{e}$, and $1.6 R_{e}$. Figure 4 demonstrates that the FIRE and MOSFIRE spectra agree well, and we therefore combine them in the bottom of the panel to search for gradients in Balmer line strength. No variation is apparent, even though the spectra would be sensitive to an age difference of $\simeq 200$ Myr. Thus, the last star formation event in RG1M0150 was not highly centrally concentrated.

\subsection{Ionized Gas}

We detect emission in the $[\mathrm{N}$ II] $\lambda \lambda 6550,6585$ and $\mathrm{H} \alpha$ lines (see Figure 3). Weak $\mathrm{H} \alpha$ emission with a modest equivalent width of $\mathrm{EW}_{\mathrm{H} \alpha}=6 \pm 2 \AA$ (rest frame) is evident only after subtracting the underlying stellar absorption. [N $\mathrm{NI}$ ] is much stronger $\left(\mathrm{EW}_{[\mathrm{NII}] \lambda 6585}=22 \pm 2 \AA\right)$. The very high ratio log $([\mathrm{N}$ II $] \lambda 6585 / \mathrm{H} \alpha)=0.6 \pm 0.2$ exceeds that attainable through photoionization by a starburst (e.g., Kewley et al. 2001). The main ionizing source is therefore not OB stars, a conclusion consistent with the low star formation rate inferred from the stellar continuum. Although other bright emission line diagnostics are not available (e.g., [O II] and [O III] are inaccessible due to atmospheric opacity), similarly high $[\mathrm{N} \mathrm{II}] / \mathrm{H} \alpha$ ratios are sometimes seen in red sequence and post-starburst galaxies at lower redshift that usually have LINER-type spectra (e.g., Yan et al. 2006; Lemaux et al. 2010). The [N II] 26585 emission in RG1M0150 has a roughly constant EW across the galaxy, which suggests (but does not prove) that the main ionizing source is distributed similarly to the stars (e.g., post-AGB stars or shocks) rather than an active galactic nucleus (AGN). X-ray, radio, and mid/far-IR observations could clarify this by testing for the presence of an AGN or obscured star formation.

\section{STELLAR KINEMATICS: ROTATION IN A QUIESCENT $z>2$ GALAXY}

Stellar kinematics were measured using the $H$-band $\left(\lambda_{\text {rest }}=4060-4930 \AA\right)$ spectra within the spatial apertures described in Section 3.2. We use the ppxf code (Cappellari \& Emsellem 2004) and allow a linear combination of Bruzual \& Charlot (2003) simple stellar populations as the template.

\subsection{Velocity Dispersion}

We first determine the velocity dispersion $\sigma_{e}$ within the aperture encompassing $R_{e}$ (see Section 3.2). This is simply the second moment of the line-of-sight velocity distribution and includes both random and streaming motions. The FIRE and MOSFIRE spectra yield consistent estimates of $\sigma_{e, \text { stars }}=288 \pm 27 \mathrm{~km} \mathrm{~s}^{-1}$ and $254 \pm 25 \mathrm{~km} \mathrm{~s}^{-1}$, respectively, which we average to obtain $\sigma_{e \text {, stars }}=271 \pm 18 \mathrm{~km} \mathrm{~s}^{-1}$. Tests of several systematic uncertainties revealed that sensitivity to template construction and the inclusion or exclusion of Balmer lines dominate the error budget, contributing $\pm 38 \mathrm{~km} \mathrm{~s}^{-1}$. Combining the uncertainties in quadrature yields $\sigma_{e, \text { stars }}=271 \pm 42 \mathrm{~km} \mathrm{~s}^{-1}$, which is comparable to that measured for the ionized gas in the same aperture, $\sigma_{e, \mathrm{gas}}=255 \pm 32 \mathrm{~km} \mathrm{~s}^{-1}$.

\subsection{Rotation and Dynamical Model}

Although our spectra are insufficient for resolved measurements of $\sigma$, we do have sufficient signal to measure velocities in our five spatial bins. This the first such measurement derived from stellar absorption features at redshifts beyond the $z \sim 1$ study of van der Wel \& van der Marel (2008). Figure 4 (right panel) shows that all 4 independent measurements on the southern half of the arc are redshifted, while all 4 measurements on the northern half are blueshifted with respect to $z_{\text {sys }}=2.6356 \pm 0.0002$ measured in the central spatial bin. The presence of rotation with the same directional sense in independent data sets gives us significantly higher confidence in its reality.

To compare the rotational velocity of RG1M0150 with other samples, we must account for observational effects, particularly the seeing, slit width, and binning. We do this by constructing a two-integral (semi-isotropic) oblate Jeans dynamical model. We assume mass follows the light distribution described by our Sérsic model. The free parameters are $M_{\mathrm{dyn}}$, the rotation parameter $k$ (Satoh 1980), and the inclination. (See van der Wel \& van der Marel 2008 for a quite similar approach.) The dynamical model is constructed in the source plane, then lensed, convolved by the seeing, and binned like the data. The model is constrained by the rotation points (Figure 4) and $\sigma_{e \text {,stars. }}$ Fortuitously, the slit PA of -7.5 , which is aligned with the direction of magnification, is close to the major axis PA $\left(2^{\circ} \pm 14^{\circ}\right)$.

The inferred dynamical mass is $\log M_{\text {dyn }}=11.24 \pm 0.14$ when marginalizing over the unknown inclination $0<\cos i<\cos i_{\min }$. Here we choose $i_{\min }=29^{\circ}$ because it corresponds to a maximum intrinsic ellipticity $e_{\text {int }}=0.8$. Although the intrinsic $(V / \sigma)_{\text {int }}$ is sensitive to inclination, the projected value that we measure is not. The inferred $M_{\text {dyn }}$ is also insensitive to inclination except when the galaxy is close to face-on (this result is generic in Jeans dynamical modeling, e.g., van der Marel 1991). In particular, when fixing $i=i_{\text {min }}$ we find that that the inferred $M_{\text {dyn }}$ would increase significantly by 0.16 dex. Adopting any inclination $i>32^{\circ}$ that is even slightly larger, however, affects $M_{\text {dyn }}$ by $<0.05$ dex.

Adopting a local virial calibration $\log M_{\mathrm{vir}}=$ $\log 5 \sigma_{e}^{2} R_{e} / G=11.19 \pm 0.12$ would have given a slightly smaller mass. $M_{\mathrm{dyn}}$ is smaller than, but consistent with, our estimate of $M_{*}$. However, some tension would arise had we adopted a Salpeter-type initial mass function. It will be interesting to evaluate this further with larger samples of lensed galaxies.

Observational effects modify the observed rotation significantly, as Figure 4 demonstrates. Our modeling allows us to account for these. The best-fitting model has a maximum projected rotation velocity $V_{\max }=189 \pm 34 \mathrm{~km} \mathrm{~s}^{-1}$ and $V / \sigma=0.70 \pm 0.21$. This model adopts the classic definition of $V / \sigma$ based on $V_{\max }$ and $\sigma_{0}$ averaged within $R_{e} / 2$ (e.g., Davies et al. 1983).

\section{DISCUSSION}

A central question in the evolution of ETGs is the extent to which the quenching of star formation and the transformation of stellar structure, morphology, and kinematics proceed 


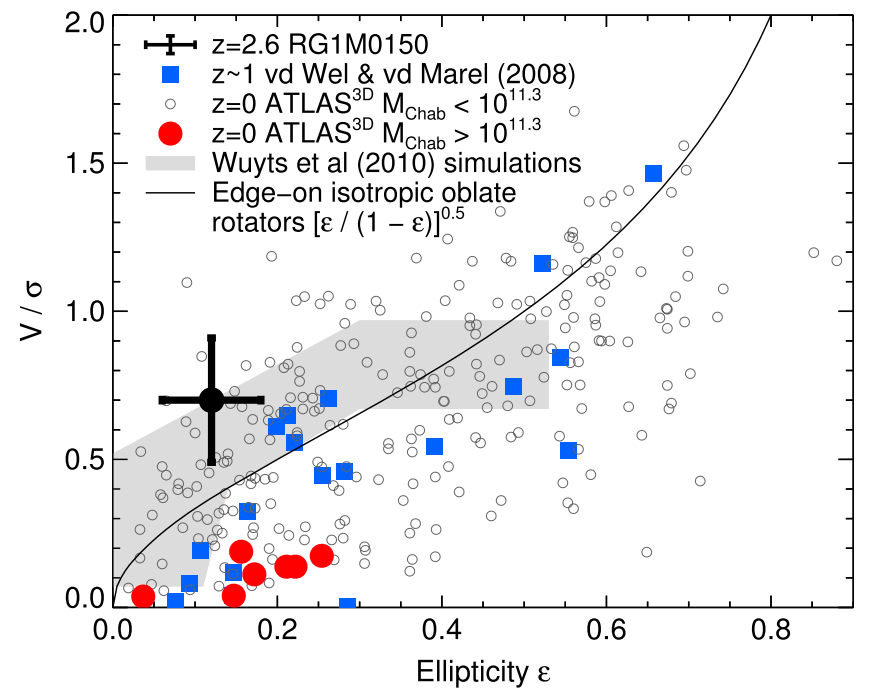

Figure 5. Rotational support in RG1M0150 is compared to observations of ETGs at $z \sim 1$ (van der Wel \& van der Marel 2008) and $z \sim 0$ (Emsellem et al. 2011, ATLAS ${ }^{3 D}$ ). The solid line is the Binney (1978) curve for isotropic oblate rotators. The gray region approximately encompasses the simulated high- $z$ merger remnants from Wuyts et al. (2010).

together (e.g., in one major merger episode) or in several distinct events. The presence of compact quiescent galaxies at high redshift demonstrates that growth of the stellar distribution proceeds long after quenching. Recent examinations of the evolving ellipticities of quiescent galaxies suggest that these early, compact systems were also more disk-like (van der Wel et al. 2011; Chang et al. 2013). The key test is a direct measurement of rotation, presented in this Letter for the first time.

Figure 5 compares the rotational support of RG1M0150 with that of local ETGs in the classic $(\epsilon, V / \sigma)$ diagram. $^{6}$ RG1M0150 has a high $V / \sigma$ for its modest observed ellipticity that places it on the upper envelope of local ETGs. Although we cannot determine the inclination and deproject $V / \sigma$ with current data, it is possible that the edge-on rotation velocity is significantly higher.

Numerical simulations of galaxy mergers require high gas fractions of $\sim 40 \%$ (Wuyts et al. 2010) to produce compact remnants such as RG1M0150. These simulations generally find that a significant amount of angular momentum is retained and suggest that rotation will be more significant in quiescent galaxies at higher redshift, when mergers were more dissipative. Wuyts et al. (2010) predicted that quiescent $z \sim 2$ galaxies would fall on the upper envelope of local ETGs in the $(\epsilon, V / \sigma)$ diagram. Intriguingly, we find that RG1M0150 indeed lies close in Figure 5 to their simulated merger remnants, which are compact systems with $M_{*} \sim 10^{11} M_{\odot}$ and $R_{e} \sim 1 \mathrm{kpc}$ comparable to typical $z \simeq 2$ quiescent galaxies.

Simulations by Zolotov et al. (2015) predict that the gas in compact quiescent galaxies and their immediate star-forming progenitors is kinematically colder than the stars, i.e., has higher $V / \sigma$. This causes the projected $\sigma_{e, \text { stars }} / \sigma_{e, \text { gas }}$ to be $\sim 1$ for an edge-on view but progressively higher for lower inclinations. Supporting this, Barro et al. (2015) measured

\footnotetext{
6 Using the ATLAS ${ }^{3 \mathrm{D}}$ kinematic maps, we determined that their adopted definition $(V / \sigma)_{e}$ (Equation (10) of Cappellari et al. 2007) is lower than ours by an average factor of 1.53. We apply this correction to place the data in Figure 5 on a uniform scale.
}

$\sigma_{\text {stars }} / \sigma_{\text {gas }}=1.7 \pm 0.5$ in a quenching compact galaxy at $z=1.7$. We find that the stellar and gas line widths are closer in $\mathrm{RG} 1 \mathrm{M} 0150$, with $\sigma_{e \text {, stars }} / \sigma_{e \text {,gas }}=1.1 \pm 0.2$, but larger samples will be needed to test this hypothesis due to the strong inclination dependence.

Nearly all local ETGs with $M_{*}>10^{11.3} M_{\odot}$ (scaled to a Chabrier IMF) are "slow rotators" with $V / \sigma$ much smaller than RG1M0150 (red points in Figure 5). Our best estimate for the stellar mass of RG1M0150 already places it within this range. Even allowing a somewhat smaller mass (e.g., our $M_{\text {dyn }}$ ), it is quite likely that RG1M0150 will evolve into a slow rotator: empirical estimates based on number density arguments (e.g., Muzzin et al. 2013; Patel et al. 2013) imply that RG1M0150 will grow in stellar mass by $0.2-0.4$ dex by $z=0$. Stellar populations in such ultra-massive galaxies today are almost exclusively very old (e.g., McDermid et al. 2015), so significant later star formation is unlikely. If RG1M0150 is typical, this suggests that the progenitors of giant ellipticals are still rotating significantly after they have been quenched and must reduce their net angular momentum later. A sequence of mostly dry mergers, suspected of driving the observed $\simeq 4 \times$ growth in effective radius, might provide a physical explanation.

General conclusions about galaxy formation obviously cannot be drawn from RG1M0150 alone. Fortunately, a larger sample of lensed compact galaxies at $z \sim 2$ (mostly at lower masses) is within reach and provides the most feasible route toward resolving the stellar populations and dynamics of these intriguing objects.

We thank Dan Kelson for expertise reducing the FourStar data and insightful discussions. It is a pleasure to acknowledge helpful conversations with Adam Muzzin and Sune Toft, as well as the referee for a prompt and thoughtful report. The authors acknowledge the very significant cultural role that the summit of Mauna Kea has always had within the indigenous Hawaiian community. We are most fortunate to have the opportunity to conduct observations from this mountain.

\section{REFERENCES}

Barro, G., Faber, S. M., Dekel, A., et al. 2015, ApJ, submitted (arXiv:1503.07164)

Barro, G., Trump, J. R., Koo, D. C., et al. 2014, ApJ, 795, 145

Belli, S., Newman, A. B., \& Ellis, R. S. 2015, ApJ, 799, 206

Binney, J. 1978, MNRAS, 183, 501

Bruzual, G., \& Charlot, S. 2003, MNRAS, 344, 1000

Calzetti, D., Armus, L., Bohlin, R. C., et al. 2000, ApJ, 533, 682

Cappellari, M., \& Emsellem, E. 2004, PASP, 116, 138

Cappellari, M., Emsellem, E., Bacon, R., et al. 2007, MNRAS, 379, 418

Chabrier, G. 2003, PASP, 115, 763

Chang, Y.-Y., van der Wel, A., Rix, H.-W., et al. 2013, ApJ, 762, 83

Cox, T. J., Dutta, S. N., Di Matteo, T., et al. 2006, ApJ, 650, 791

Davies, R. L., Efstathiou, G., Fall, S. M., Illingworth, G., \& Schechter, P. L. 1983, ApJ, 266, 41

Ebeling, H., Edge, A. C., \& Henry, J. P. 2001, ApJ, 553, 668

Elíasdóttir, Á., Limousin, M., Richard, J., et al. 2007, arXiv:0710.5636

Emsellem, E., Cappellari, M., Krajnović, D., et al. 2011, MNRAS, 414, 888

Geier, S., Richard, J., Man, A. W. S., et al. 2013, ApJ, 777, 87

Hopkins, P. F., Cox, T. J., Dutta, S. N., et al. 2009, ApJS, 181, 135

Jullo, E., Kneib, J.-P., Limousin, M., et al. 2007, NJPh, 9, 447

Kelson, D. D., Williams, R. J., Dressler, A., et al. 2014, ApJ, 783, 110

Kewley, L. J., Dopita, M. A., Sutherland, R. S., Heisler, C. A., \& Trevena, J. 2001, ApJ, 556, 121

Kneib, J. P., Mellier, Y., Fort, B., \& Mathez, G. 1993, A\&A, 273, 367

Lemaux, B. C., Lubin, L. M., Shapley, A., et al. 2010, ApJ, 716, 970

McDermid, R. M., Alatalo, K., Blitz, L., et al. 2015, MNRAS, 448, 3484 
McLean, I. S., Steidel, C. C., Epps, H. W., et al. 2012, Proc. SPIE, 8446, $84460 \mathrm{~J}$

Muzzin, A., Labbé, I., Franx, M., et al. 2012, ApJ, 761, 142

Muzzin, A., Marchesini, D., Stefanon, M., et al. 2013, ApJ, 777, 18

Newman, A. B., Ellis, R. S., Andreon, S., et al. 2014, ApJ, 788, 51

Newman, A. B., Ellis, R. S., Bundy, K., \& Treu, T. 2012, ApJ, 746, 162

Newman, A. B., Ellis, R. S., \& Treu, T. 2015, ApJ, submitted (arXiv:1503.05282)

Newman, A. B., Treu, T., Ellis, R. S., et al. 2013, ApJ, 765, 24

Patel, S. G., van Dokkum, P. G., Franx, M., et al. 2013, ApJ, 766, 15

Persson, S. E., Murphy, D. C., Smee, S., et al. 2013, PASP, 125, 654

Richard, J., Smith, G. P., Kneib, J.-P., et al. 2010, MNRAS, 404, 325

Robertson, B., Cox, T. J., Hernquist, L., et al. 2006, ApJ, 641, 21

Satoh, C. 1980, PASJ, 32, 41

Simcoe, R. A., Burgasser, A. J., Schechter, P. L., et al. 2013, PASP, 125, 270
Skelton, R. E., Whitaker, K. E., Momcheva, I. G., et al. 2014, ApJS, 214, 24 Toft, S., Smolčić, V., Magnelli, B., et al. 2014, ApJ, 782, 68

Trujillo, I., Feulner, G., Goranova, Y., et al. 2006, MNRAS, 373, L36

Vacca, W. D., Cushing, M. C., \& Rayner, J. T. 2003, PASP, 115, 389

van der Marel, R. P. 1991, MNRAS, 253, 710

van der Wel, A., Franx, M., van Dokkum, P. G., et al. 2014, ApJ, 788, 28

van der Wel, A., Rix, H.-W., Wuyts, S., et al. 2011, ApJ, 730, 38

van der Wel, A., \& van der Marel, R. P. 2008, ApJ, 684, 260

van Dokkum, P. G., Nelson, E. J., Franx, M., et al. 2015, ApJ, in press (arXiv:1506.03085)

van Dokkum, P. G., Whitaker, K. E., Brammer, G., et al. 2010, ApJ, 709, 1018

Wellons, S., Torrey, P., Ma, C.-P., et al. 2015, MNRAS, 449, 361

Wuyts, S., Cox, T. J., Hayward, C. C., et al. 2010, ApJ, 722, 1666

Yan, R., Newman, J. A., Faber, S. M., et al. 2006, ApJ, 648, 281

Zolotov, A., Dekel, A., Mandelker, N., et al. 2015, MNRAS, 450, 2327 